\title{
Development of Methods of Asymptotic Analysis of Transition Layers in Reaction-Diffusion-Advection Equations: Theory and Applications
}

\author{
N. N. Nefedov ${ }^{a}$ \\ ${ }^{a}$ Faculty of Physics, Lomonosov Moscow State University, Moscow, 119991 Russia \\ e-mail:nefedov@phys.msu.ru
}

Received March 25, 2021; revised March 25, 2021; accepted August 4, 2021

\begin{abstract}
This work presents a review and analysis of modern asymptotic methods for analysis of singularly perturbed problems with interior and boundary layers. The central part of the work is a review of the work of the author and his colleagues and disciples. It highlights boundary and initial-boundary value problems for nonlinear elliptic and parabolic partial differential equations, as well as periodic parabolic problems, which are widely used in applications and are called reaction-diffusion and reaction-diffusion-advection equations. These problems can be interpreted as models in chemical kinetics, synergetics, astrophysics, biology, and other fields. The solutions of these problems often have both narrow boundary regions of rapid change and inner layers of various types (contrasting structures, moving interior layers: fronts), which leads to the need to develop new asymptotic methods in order to study them both formally and rigorously. A general scheme for a rigorous study of contrast structures in singularly perturbed problems for partial differential equations, based on the use of the asymptotic method of differential inequalities, is presented and illustrated on relevant problems. The main achievements of this line of studies of partial differential equations are reflected, and the key directions of its development are indicated.
\end{abstract}

Keywords: singularly perturbed problems, asymptotic methods, boundary and interior layers, fronts, reaction-diffusion-advection equations, contrast structures, balanced nonlinearity, differential inequalities, Lyapunov asymptotic stability, asymptotic solution of inverse problems

DOI: $10.1134 / \mathrm{S} 0965542521120095$

\section{INTRODUCTION}

Presently, one of the topical problems in the singular perturbation theory is the study of nonlinear singularly perturbed partial differential equations, the solutions of which have boundary and interior layers. Such equations are of great interest both in the qualitative theory of differential equations and in many applied problems. In particular, such equations arise in mathematical models of processes of the reaction-diffusion and reaction-diffusion-advection type in chemical kinetics, synergetics, astrophysics, biology, and other fields, where the processes under study are characterized by narrow boundary regions of rapid changes in process parameters or by sharp interior layers of various types (contrast structures), stationary or moving (fronts). For an adequate mathematical description of such processes, it is necessary to develop new asymptotic methods for studying nonlinear singularly perturbed problems, the studies of which (initial value problems with small parameters at higher derivatives) began quite a long time ago. The direction of research presented in this work is the development of the deep ideas of the works of A.N. Tikhonov (see [1-3]), who laid the foundations of the modern singular perturbation theory. These works, which combined the singular perturbation theory with the qualitative theory of differential equations and Lyapunov's stability theory and were continued and developed in the works of A.B. Vasilieva, V.F. Butuzov, and their students and followers (see the book by Vasilieva and Butuzov [4]), to this day form the language of the singular perturbation theory, despite the significantly complicated problems under consideration. 
Various areas of the theory of nonlinear singularly perturbed problems are being intensively developed both in our country and abroad. This is evidenced by a number of international conferences held in recent years and devoted to the singular perturbation theory, as well as conferences devoted to interior layers. In our country, these studies are carried out by the school of V.P. Maslov and S.Yu. Dobrokhotov; disciples of S.A. Lomov, S.A. Kashchenko, S.D. Glyzin, and many others. These works have been and are being actively pursued in the USA, European countries, Japan, and China (P. Fife, B. McLeod, P. Bates, N. Alikakos, Danielle Hilhorst, B. Fidler, L. Recke, K. Scheider, P. Szmolyan, H. Motano, H. Morita, K. Sakamoto, Vimura, Nishiura, and others). The results of these and other specialists are presented in the proceedings of the conference "Patterns of Dynamics 2016", held in Berlin, and in a special issue of the journal "Discrete and continuous dynamical systems," 37 (2), February 2017 (see [5]), dedicated to memory of Paul Fife.

The current state of the asymptotic analysis of singularly perturbed problems with boundary and inner layers can be found in the review [6]. A number of interesting results developing the studies of problems with interior and boundary layers were obtained in [7-9]. Currently, there is a significant increase in interest in singularly perturbed delay equations, which is associated with the consideration of more complex models important for applications (see, e.g., $[10,11]$ and references therein). One of the most important applications of asymptotic analysis of singularly perturbed problems is the development of a theory of numerical methods for problems with transition layers. A significant contribution to the development of this direction was made in the works of A.M. Il'in, N.S. Bakhvalov, G.I. Shishkin, and N.V. Kopteva and Irish mathematicians M. Stynes, E. O'Riordan, and others. The results of these works can be found in the review [12]. A number of interesting results concerning theory and their application for the numerical solution of singularly perturbed problems with transition layers were obtained in [13-18]. Note that this actively developing area is based on the results of an asymptotic analysis of the works presented in this review.

In this paper, we present a development of the well-known method of boundary functions for constructing asymptotics for solutions of various classes of problems with interior layers. The main ideas are demonstrated on new classes of singularly perturbed problems, including problems on the motion of fronts and studies of stable stationary or periodic interior layers in multidimensional problems. These results are a further development of our studies of contrast structures, which were published in review articles [19-22].

Contrast structures are usually understood as solutions with interior layers of nonlinear singularly perturbed equations. Such solutions have been studied for a long time. The first results in this direction were obtained by A.B. Vasilieva back in the early 1970s for a two-point boundary value problem

$$
\begin{gathered}
\varepsilon^{2} \frac{d^{2} u}{d x^{2}}-f(u, x)=0, \quad x \in(0,1), \\
u(0, \varepsilon)=u^{(0)}, \quad u(1, \varepsilon)=u^{(1)} .
\end{gathered}
$$

It was proved that there exists a solution that, for small $\varepsilon$, is close to the root $u=\varphi_{1}(x)$ of the degenerate equation $f(u, x)=0$ to the left of a certain point $x_{0}$ and close to the root $u=\varphi_{2}(x)$ to the right of $x_{0}$. In this case, the point $x_{0}$ was not known in advance, but was determined in the course of constructing the asymptotics. Such solutions are now commonly referred to as step-type contrast structures. An example of nonlinearity for which solutions of the above type exist is $f(u, x)=(u-\varphi(x))\left(u^{2}-1\right)$. In this case, $\varphi_{1}(x)=-1, \varphi_{2}(x)=1$, and $x_{0}$ is a simple root of the equation $\varphi(x)=0$. These results were extended to some classes of quasilinear equations that are one-dimensional in the space variable. Methods for constructing the asymptotics of such classes of problems and the substantiation of the asymptotics constructed are contained in the book by A.B. Vasilieva and V.F. Butuzov (see [4]). A significant contribution to the development of asymptotic methods for analysis of nonlinear partial differential equations was made by the American mathematician P. Fife, communications with whom I recall with deep gratitude. He considered a multidimensional analogue of problem (1). The results of his work on boundary and interior layers for a multidimensional analogue of problem (1) (see [23, 24]) played an essential role in the development of this line of studies. It should be noted that the existence of a boundary-layer solution and a solution with an interior transition layer in $[23,24]$ was proved using methods similar to those used by A.B. Vasilieva for one-dimensional problems, while their realization in multidimensional problems is much more difficult. 
If we consider a two-dimensional analogue of problem (1),

$$
\begin{gathered}
\varepsilon^{2} \Delta u=f(u, x, \varepsilon), \quad x \in \mathscr{D} \subset R^{2}, \\
u(x, \varepsilon)=g(x), \quad x \in \partial \mathscr{D},
\end{gathered}
$$

where $\varepsilon>0$ is a small parameter and $\Delta$ is the Laplacian, the contrast structure can be defined as follows.

A step-type contrast structure is a solution $u(x, \varepsilon)$ of problem (2) that is close to two different solutions of the degenerate equation $f(u, x, 0)=0$ on opposite sides of some closed curve $\Gamma$ (the position of the curve $\Gamma$ is not known in advance, but is determined in the course of constructing the asymptotics). In the vicinity of the curve $\Gamma$, a region of rapid change in the solution arises; the solution in this region is called an interior transition layer. In [25, 26], a new method was proposed for proving the existence of solutions of singularly perturbed partial differential equations, called the asymptotic method of differential inequalities. This method turned out to be effective, and both the general scheme of this method was developed (see, e.g., [27-29]) and its application to other classes of singularly perturbed problems: initial-boundary value problems for parabolic equations in describing solutions with moving interior layers (fronts), periodic parabolic boundary value problems, boundary and initial-boundary value problems for some classes of integro-differential equations, and some classes of systems.

Another class of singularly perturbed problems discussed in this paper are problems with discontinuous nonlinearities (sources or advective coefficients), which simulate transition layers in the contact region of different media, as well as phase transitions. The paper presents new results concerning the existence of solutions of this class of problems, their asymptotics, and stability.

The study of the problems considered is based on the asymptotic method of differential inequalities. The main idea of this approach is the construction of lower and upper solutions of the problem using formal asymptotics. For all the problems considered, the existence of solutions is proved, the accuracy of the asymptotics is estimated, and their stability is analyzed.

The results presented in this paper form the basis for further development of methods for asymptotic analysis of new classes of nonlinear singularly perturbed problems for partial differential equations, which are widely used as mathematical models in many important applications. The development of asymptotic methods for studying complex nonlinear mathematical models with sharp transition layers will allowalong with solving important mathematical questions of existence, stability, and asymptotic approximation of solutions - to develop efficient numerical methods for solving these classes of problems, as well as new concepts of solving inverse problems based on asymptotic analysis, analyze conditions of destruction and sharpening of solutions, as well as the application of the results obtained in a number of applications: simulation of contrast structures in the studies of problems in biophysics, ecology, genetics, nonlinear wave theory, and other applications.

It should be noted that this review does not claim to be complete. This paper highlights the line of studies pursued by the scientific school to which the author belongs and the method to the development of which he is directly related.

\section{BASIC IDEAS OF THE ASYMPTOTIC METHOD OF DIFFERENTIAL INEQUALITIES}

In our works, a general scheme for a rigorous study of contrast structures in singularly perturbed problems for partial differential equations was developed based on the asymptotic method of differential inequalities. Below, for simplicity of presentation, we will explain this scheme on the example of problem (2), without specifying the requirements under which its implementation is possible. As noted in Introduction, this scheme consists of three main stages: constructing the formal asymptotics of the contrast structure, modifying this asymptotics to construct the upper and lower solutions of the problem, and using the lower and upper solutions constructed to prove the Lyapunov asymptotic stability of stationary or periodic solutions in the corresponding classes of problems. These steps will be outlined below in this section.

\subsection{Construction of Formal Asymptotics}

Step-type contrast structures in problem (2) and other similar problems are studied under the assumption that the nonlinearity $f$ is bistable, i.e., has two stable roots $\varphi^{( \pm)}(x)$ and an unstable root $\varphi^{(0)}(x)$ such 
that $\varphi^{(-)}(x)<\varphi^{(0)}(x)<\varphi^{(+)}(x)$ for $x \in \bar{D}$. The position of the transition curve $\Gamma_{\varepsilon}$ between the roots $\varphi^{(-)}$ and $\varphi^{(+)}$is usually determined by the condition

$$
u(x, \varepsilon)=\varphi^{(0)}(x), \quad x \in \Gamma_{\varepsilon}
$$

(i.e., $\Gamma_{\varepsilon}$ is defined as the projection of the curve of intersection between the solution $u(x, \varepsilon)$ and root $\varphi^{(0)}(x)$ onto the region $\left.\mathscr{D}\right)$.

Let $\Gamma_{0}$ be some closed sufficiently smooth curve lying in $\mathscr{D}$. In the neighborhood of $\Gamma_{0}$, we introduce local coordinates $r$ and $y$, where $r$ is the distance from a point from the neighborhood to the curve $\Gamma_{0}$ along the normal to the curve, taken with the "+" sign if the point lies inside the curve and with the "-" sign if the point lies in the exterior of the curve, and $y$ is the coordinate of the point on the curve from which the specified normal issues. The equation of the curve $\Gamma_{\varepsilon}$ is sought in the form

$$
r=R(y, \varepsilon)=\varepsilon R_{1}(y)+\varepsilon^{2} R_{2}(y)+\ldots .
$$

The curve $\Gamma_{0}$ and functions $R_{i}(y)$ are determined in the course of constructing the asymptotics.

The curve $\Gamma_{\varepsilon}$ divides $\overline{\mathscr{D}}$ into regions $\overline{\mathscr{D}}^{(+)}$and $\overline{\mathscr{D}}^{(-)}$, internal and external to it, respectively.

In the region $\overline{\mathscr{D}}^{(+)}$, we consider the problem

$$
\begin{gathered}
L_{\varepsilon} u \equiv \varepsilon^{2} \Delta u-f(u, x, \varepsilon)=0, \quad x \in \overline{\mathscr{D}}^{(+)}, \\
u(x, \varepsilon)=\varphi^{(0)}(x), \quad x \in \Gamma_{\varepsilon} .
\end{gathered}
$$

We construct the asymptotics $U^{(+)}$of problem (4) by the method of boundary functions (see [4, 20]) in the form

$$
U^{(+)}=\sum_{i=0}^{n+1} \varepsilon^{i}\left(\bar{u}_{i}^{(+)}+Q_{i}^{(+)}\right),
$$

where $\bar{u}_{i}^{(+)}$and $Q^{(+)}$denote the regular and boundary-layer (near $\Gamma_{\varepsilon}$ ) parts of the asymptotics.

In the region $\overline{\mathscr{D}}^{(-)}$, we consider the problem

$$
\begin{gathered}
L_{\varepsilon} u \equiv \varepsilon^{2} \Delta u-f(u, x, \varepsilon)=0, \quad x \in \overline{\mathscr{D}}^{(-)}, \\
u(x)=g(x), \quad x \in \partial D, \quad u(x, \varepsilon)=\varphi^{(0)}(x), \quad x \in \Gamma_{\varepsilon} .
\end{gathered}
$$

The asymptotics $U^{(-)}$of problem (6) is constructed by analogy with (5) in the form

$$
U^{(-)}=\sum_{i=0}^{n+1} \varepsilon^{i}\left(u_{i}^{(-)}+Q_{i}^{(-)}+\Pi_{i}\right),
$$

where $u_{i}^{(-)}$denotes the regular part of the asymptotics and $\Pi$ and $Q^{(-)}$are the boundary-layer parts of the asymptotics near the boundary $\partial \mathscr{D}$ and $\Gamma_{\varepsilon}$, respectively. The main terms of the regular parts of the asymptotics are chosen in the form $\bar{u}_{0}^{( \pm)}=\varphi^{( \pm)}$, and the functions $Q_{i}^{( \pm)}$are used to describe the transition layer near $\Gamma_{\varepsilon}$.

By construction (see $[4,20]$ ), asymptotics (5) and (7) satisfy problems (4) and (6) with a residual up to $O\left(\varepsilon^{n+2}\right)$, in particular,

$$
\begin{gathered}
L_{\varepsilon} U^{(+)}=L_{0 R} \bar{u}_{0}^{(+)}+L_{0 I L} Q_{0}^{(+)} \\
+\sum_{i=1}^{n+1} \varepsilon^{i}\left(\left(L_{R}^{(+)} \bar{u}_{i}^{(+)}+\bar{f}_{i}^{(+)}\right)+\left(L_{I L}^{(+)} Q_{i}^{(+)}+q_{i}^{(+)}\right)\right)+O\left(\varepsilon^{n+2}\right)=O\left(\varepsilon^{n+2}\right),
\end{gathered}
$$

since all the terms in the sum, except the last one, are equal to zero by virtue of the equations for the coefficients of the asymptotics; in particular, $L_{0 R} \bar{u}_{0}^{(+)}=0$ and $L_{0 I L} Q_{0}^{(+)}=0$ are nonlinear equations for determining the principal terms of asymptotics (5). The remaining coefficients of the asymptotics are deter- 
mined from linear equations with the help of invertible operators: the operator $L_{R}^{(+)}$, generating the regular part of the asymptotics, and the operator $L_{I L}^{(+)}$, generating the boundary-layer part of the asymptotics. A representation similar to (8) also holds for problem (6):

$$
\begin{gathered}
L_{\varepsilon} U^{(-)}=L_{0 R} \bar{u}_{0}^{(-)}+L_{0 I L} Q_{0}^{(-)}+L_{0 B L} \Pi_{0} \\
+\sum_{i=1}^{n+1} \varepsilon^{i}\left(\left(L_{R}^{(-)} \bar{u}_{i}^{(-)}+\bar{f}_{i}^{(-)}\right)+\left(L_{I L}^{(-)} Q_{i}^{(+)}+q_{i}^{(-)}\right)+\left(L_{B L} \Pi_{i}-\pi_{i}\right)\right) \\
+O\left(\varepsilon^{n+2}\right)=O\left(\varepsilon^{n+2}\right) .
\end{gathered}
$$

In this case, $L_{0 B L} \Pi_{0}=0$ is a nonlinear equation for determining the leading term of the boundary-layer part of the asymptotics near the boundary $\partial \mathscr{D}$ and the subsequent boundary functions are determined from linear equations by virtue of the invertible operator $L_{B L}$. The rest of the terms in (9) are completely analogous to the corresponding terms in (8).

By virtue of the boundary conditions on the curve $\Gamma_{\varepsilon}$ in problems (4) and (6), the asymptotics $\bar{U}^{(+)}$and $\bar{U}^{(-)}$are joined by continuity (the $C$-matching condition is satisfied) on the curve $\Gamma_{\varepsilon}$. This curve (i.e., the curve $\Gamma_{0}$ ) and the coefficients $R_{i}$ in expansion (3) are determined from the condition of $C^{1}$-matching of the asymptotics $\bar{U}^{(+)}$and $\bar{U}^{(-)}$on $\Gamma_{\varepsilon}$ :

$$
\varepsilon \frac{\partial \bar{U}^{(-)}}{\partial r}=\varepsilon \frac{\partial \bar{U}^{(+)}}{\partial r}, \quad x \in \Gamma_{\varepsilon}
$$

considered in the appropriate approximation in $\varepsilon$. In this case, $\Gamma_{0}$ is usually found from a nonlinear problem. To determine $R_{i}$, the linear equations are obtained in the form

$$
A^{\Gamma} R_{i}=H_{i},
$$

where $A^{\Gamma}$ is an invertible operator and $H_{i}$ is a function known at each step.

This algorithm will be demonstrated in more detail with examples in the following sections.

\subsection{Asymptotic Method of Differential Inequalities}

We developed the asymptotic method of differential inequalities for those classes of singularly perturbed problems where the principle of comparison of elliptic boundary value problems, parabolic initialboundary value problems, and time-periodic parabolic boundary value problems, as well as problems for integro-differential equations, works. Basic comparison theorems for nonlinear equations, based on the principle of maximum or monotonicity of linearized operators, originate from the well-known S.A. Chaplygin's comparison theorems for initial-boundary value problems and Nagumo's theorems for boundary value problems and are one of the most important tools for studying nonlinear partial differential equations. These theorems can be found in a number of articles and monographs (see, e.g., [30] and references therein). A significant contribution to the development of the comparison principle was made by M.A. Krasnosel'skii, M.G. Krein, S.I. Pokhozhaev, H. Amann, P. Hess, and D.J. Sattinger (see [31-37]). These ideas were further developed in the works for special cases (see [38-44]).

The main idea of the asymptotic method of differential inequalities is the use of a modified formal asymptotics to construct comparison functions: lower and upper solutions; in this case, the main requirement for the implementation of this approach is the monotonicity of the asymptotics-generating operators linearized on the zeroth order. This approach was proposed in [25, 26] and determined a strategy for proving the existence and estimating the accuracy of the asymptotics constructed. In [27], this method was extended to periodic parabolic boundary value problems. In that work, a fairly effective method was proposed for proving the asymptotic stability of periodic solutions, which was then transferred to the analysis of the asymptotic stability of stationary solutions of initial-boundary value problems for reaction-diffusion-type parabolic equations and then generalized to some more complex classes of reaction-diffusionadvection-type quasilinear equations (see $[29,45]$ ). In recent years, this approach has been extended to reaction-diffusion-advection problems with discontinuous nonlinearities and sources (see [46] and references therein). An essential development of the method was the results on the asymptotic analysis of boundary and interior layers in various problems for integro-differential equations (see [28], as well as [47] and references therein). 
For the convenience of presentation, we recall the well-known concepts and facts concerning problem (2).

Definition. Functions $\alpha(x, \varepsilon)$ and $\beta(x, \varepsilon)$ are called lower and upper solutions of problem (2) if they satisfy the following conditions:

(i) $\quad L_{\varepsilon} \alpha(x, \varepsilon):=\varepsilon \Delta \alpha(x, \varepsilon)-f(\alpha(x, \varepsilon), x, \varepsilon) \geq 0$, $L_{\varepsilon} \beta(x, \varepsilon) \leq 0, \quad x \in \mathscr{D}$,

(ii) $\alpha(x, \varepsilon) \leq g(x) \leq \beta(x, \varepsilon), \quad x \in \partial \mathscr{D}$.

It is known (see, e.g., [30]) that, if there exist ordered lower and upper solutions of problem (2), i.e.,

$$
\alpha(x, \varepsilon) \leq \beta(x, \varepsilon), \quad x \in \overline{\mathscr{D}},
$$

then problem (2) has a solution $u(x, \varepsilon)$, satisfying the inequalities

$$
\alpha(x, \varepsilon) \leq u(x, \varepsilon) \leq \beta(x, \varepsilon), \quad x \in \overline{\mathscr{D}} \text {. }
$$

When constructing the formal asymptotics, the main requirement for the linear operators $L_{R}, L_{B L}$, and $A^{\Gamma}$ is their invertibility, whereas, when constructing the lower and upper solutions, our requirements are more stringent: we require that inequalities $L_{R} \delta \bar{u}<0, L_{B L} \delta \Pi<0$, and $A^{\Gamma} \delta R<0$ have positive solutions in the same classes of functions in which these operators are used to construct the corresponding expansions.

Then, if we add $\delta \bar{u}$ and $\delta \Pi$ to the coefficients at the terms of the order of $\varepsilon^{n+1}$ in the regular and boundarylayer parts of asymptotics (5) and (7) and add $(-\delta R(y))$ to $R_{n+1}(y)$ from Eq. (3), we will obtain the upper solution $\beta_{n+1}(x, \varepsilon)$. Similarly, by the addition of $(-\delta \bar{u}),(-\delta \Pi)$, and $\delta R$, we construct the lower solution $\alpha_{n+1}(x, \varepsilon)$. Checking according to the standard scheme (see [26]) shows that $\alpha_{n+1}(x, \varepsilon)$ and $\beta_{n+1}(x, \varepsilon)$ satisfy the relations

$$
\begin{gathered}
L_{\varepsilon} \alpha_{n+1}(x, \varepsilon)=\varepsilon^{n+1} q(x, \varepsilon), \quad L_{\varepsilon} \beta_{n+1}(x, \varepsilon)=-\varepsilon^{n+1} q(x, \varepsilon), \quad x \in \mathscr{D}, \\
\alpha_{n+1}(x, \varepsilon) \leq g(x) \leq \beta_{n+1}(x, \varepsilon), \quad x \in \partial \mathscr{D},
\end{gathered}
$$

where $q(x, 0)>0$ in $\overline{\mathscr{D}}$. In addition, by construction,

$$
\beta_{n+1}-\alpha_{n+1}=O\left(\varepsilon^{n+1}\right)>0 .
$$

It follows from (12) and (13) that $\alpha_{n+1}(x, \varepsilon)$ and $\beta_{n+1}(x, \varepsilon)$ are ordered lower and upper solutions of problem (2) and, therefore, there exists a solution $u(x, \varepsilon)$ of this problem, satisfying the inequalities

$$
\alpha_{n+1}(x, \varepsilon) \leq u(x, \varepsilon) \leq \beta_{n+1}(x, \varepsilon) .
$$

Moreover, since, by construction,

$$
\beta_{n+1}-U_{n+1}=O\left(\varepsilon^{n+1}\right)
$$

it follows from (14) and (15) that the solution of problem (2) has an estimate

$$
\left|u(x, \varepsilon)-U_{n}(x, \varepsilon)\right|=O\left(\varepsilon^{n+1}\right), \quad x \in \overline{\mathscr{D}} .
$$

Note that $\alpha_{n+1}(x, \varepsilon)$ and $\beta_{n+1}(x, \varepsilon)$ satisfying (12) are called asymptotic lower and upper solutions of order $(n+1)$.

\subsection{Asymptotic Method of Differential Inequalities in the Analysis of Lyapunov Asymptotic Stability of Stationary and Periodic Solutions}

As noted above, in [27], a fairly simple method was proposed for proving the Lyapunov asymptotic stability of periodic solutions of periodic parabolic problems and for determining the local region of stability and uniqueness of solutions with the asymptotics constructed. This method can also be used for analysis of the asymptotic stability of stationary solutions with boundary and interior layers of initial-boundary value problems for the reaction-diffusion and reaction-diffusion-advection equations. Note that the standard methods for analysis of stability, based on considering the corresponding spectral problems for equations linearized on the solution, are rather complicated and have not been significantly developed. The main difficulty of this approach is the presence of the so-called critical spectrum: eigenvalues that tend to zero as the small parameter tends to zero. This approach for partial differential equations, called the method of the singular limit of the eigenvalue problem, was first implemented in [48]. We also note 
that this work has played an important role in the development of methods for constructing asymptotics in the so-called problems with balanced nonlinearity, as well as in problems with advection, where multidimensionality plays an essential role in the structure and stability properties of the solution.

Let us illustrate the use of the method of differential inequalities on problem (2). The solution of problem (2) can be considered a stationary solution of the initial-boundary value problem

$$
\begin{gathered}
\frac{\partial u}{\partial t}=\varepsilon^{2} \Delta u-f(u, x, \varepsilon), \quad x \in \mathscr{D}, \quad t>0, \\
u(x, t, \varepsilon)=g(x), \quad x \in \partial \mathscr{D}, \quad t>0, \\
u(x, 0, \varepsilon)=u^{0}(x, \varepsilon) .
\end{gathered}
$$

It can be shown that, if the upper and lower solutions of problem (2) are sufficiently accurate ( $n$ is large enough: see the previous paragraph), then the functions $\hat{\beta}(x, t, \varepsilon)$ and $\hat{\alpha}(x, t, \varepsilon)$ (which are determined by the expressions

$$
\begin{aligned}
& \hat{\beta}(x, t, \varepsilon)=u(x, \varepsilon)+\left(\beta_{n}(x, \varepsilon)-u(x, \varepsilon)\right) e^{-\lambda t}, \\
& \hat{\alpha}(x, t, \varepsilon)=u(x, \varepsilon)+\left(\alpha_{n}(x, \varepsilon)-u(x, \varepsilon)\right) e^{-\lambda t},
\end{aligned}
$$

where $\lambda$ is a sufficiently small positive number, $\beta_{n}(x, \varepsilon)$ and $\alpha_{n}(x, \varepsilon)$ are the upper and lower solutions of problem (2), and $u(x, \varepsilon)$ is the solution of problem (2)) satisfy the corresponding differential inequalities for the parabolic initial-boundary value problem (16) (see, e.g., [30]). Then, for $\alpha_{n}(x, \varepsilon) \leq u^{0}(x, \varepsilon) \leq \beta_{n}(x, \varepsilon)$, a solution of problem (16) is $u(x, t, \varepsilon)$, which satisfies the inequalities $\hat{\alpha}(x, t, \varepsilon) \leq u(x, t, \varepsilon)<\hat{\beta}(x, t, \varepsilon)$. These inequalities with representations (17) imply the asymptotic stability of the stationary solution of problem (16) with a stability region at least $\left[\alpha_{n}(x, \varepsilon), \beta_{n}(x, \varepsilon)\right], x \in \mathscr{D}$. Moreover, $u(x, \varepsilon)$ is locally unique as a solution of problem (2) with the same uniqueness region.

\section{SOME RELEVANT PROBLEMS WITH BOUNDARY AND INTERIOR LAYERS}

In this section, we consider a number of topical problems of interest both for the singular perturbation theory and for applications. The consideration of these problems illustrates the main ideas and directions of development. One of the actively developed areas is the study of the existence and stability of stationary smooth solutions in one-dimensional and multidimensional reaction-diffusion problems with discontinuous sources and advective terms. These problems simulate transition layers at the interface between two media with different characteristics. Problems for the stationary reaction-diffusion equation on an interval and in a two-dimensional domain are considered. A specific feature of these problems is the discontinuity (of the first kind) of the reactive term in the one-dimensional case at some interior point and, in the two-dimensional case, on some smooth closed curve. This is a new previously unexplored class of problems. Methods for constructing asymptotic approximations of solutions are developed, the existence of smooth stationary solutions of reaction-diffusion equations with an interior transition layer in the vicinity of discontinuities in the one-dimensional and two-dimensional cases is proved, and conditions are obtained under which these solutions are locally unique and asymptotically Lyapunov-stable. In addition, the local region of attraction of stationary solutions is indicated. These studies are presented in $[46,49,50]$ (see also the references therein).

Another important area of studies are new classes of problems: problems with modular nonlinearities. These are new classes of mathematical problems with discontinuous advection coefficients and/or discontinuous nonlinear source terms. The main feature of these problems is that discontinuities occur at some values of the sought-for function. These problems simulate phase transitions and are initiated by the studies in nonlinear wave theory (see [51-53]). In [54, 55], initial-boundary value problems for Burgers-type equations were studied. The existence of new classes of solutions with a moving interior layer (front) was proved, conditions for a new effect in this class of problems-destruction (sharpening) of the front-were formulated, and the time for which the front moves from the initial point to the point of destruction was estimated. These works continued the study of problems for reaction-diffusion and reaction-diffusionadvection-type equations with moving interior layers (fronts). In $[47,56]$ an asymptotic approximation of the fronts was obtained in one-dimensional parabolic and integro-parabolic reaction-diffusion-type problems. These results were then transferred to quasilinear reaction-diffusion-advection-type equations with a nonlinear advection coefficient in [57-59]. Note that the consideration of moving interior layers (fronts) in bounded volumes, taking into account the spatiotemporal inhomogeneity, differs signifi- 
cantly from the studies of solutions such as traveling waves and self-similar solutions, widely presented in the literature (see, e.g., [60-62] and references therein). Similar results were obtained in the study of periodic fronts in parabolic periodic boundary value problems, including Burgers-type equations. Various problems of this class are presented in [63-68]. In these works, periodic problems, multidimensional in the spatial variable, with an interior transition layer are considered and classes of new problems are singled out in which the multidimensionality leads to new previously unexplored conditions for the existence and stability of a solution with an interior transition layer. These are problems with a balance of nonlinearity, in which the results previously obtained for the reaction-diffusion problem are generalized to problems for the reaction-diffusion-advection equation. The relationships between advection and reaction are revealed, in which advection plays a significant role in the stability of the interior transition layers.

In [69-71], a new class of nonlinear singularly perturbed problems with singularly perturbed boundary conditions of the second and third kind was investigated. The existence of stationary and time-periodic boundary-layer-type solutions was proved, and an asymptotic approximation of such solutions over a small parameter was constructed. The set of boundary conditions under which such solutions exist was investigated, and the local uniqueness and asymptotic Lyapunov stability of such solutions was established. It was shown that, unlike the analogous Dirichlet problem, where such a solution is unique, the problem under consideration can have several such solutions with individual regions of stability and local uniqueness.

Among the actively developing areas, we should single out the studies of the existence and conditions of Lyapunov stability of stationary solutions of initial-boundary value problems and periodic solutions of systems of parabolic equations with fast sources of different velocities. The asymptotics of the solution was constructed, and the asymptotic stability of the periodic solution was investigated. Systems of equations with fast sources of different velocities in the case of different quasi-monotonicity of the right-hand sides (sources) have been studied. In [72, 73], singularly perturbed systems of parabolic equations in onedimensional and two-dimensional spatial domains with periodic conditions in time were considered. The existence of a solution in the form of a periodic front was investigated. An algorithm for constructing an asymptotic approximation of the solution was presented, and a theorem of existence of a solution in the form of a periodic front, as well as its local uniqueness and asymptotic stability, was proved. A solution of this type can describe an abrupt change in the physical characteristics of some spatially inhomogeneous medium. Systems of this type are used to simulate transient processes in ecology, biophysics, chemical kinetics, semiconductor physics, and other fields. The aforementioned works are based on the development of classical methods for constructing asymptotics, proposed in the works of A.B. Vasilieva, V.F. Butuzov, and their disciples. In recent years, in the works of V.F. Butuzov, a new method was developed for constructing asymptotic approximations for the solution of singularly perturbed equations with multiple roots of degenerate equations (see [74-83] and references therein). In these works, it was shown that, in this case, the boundary and interior layers are multi-zone. The structure of the transition layers, in contrast to the cases of a simple layer, essentially depends on the type of boundary conditions. The study of the existence of solutions and their stability was based on the development of the asymptotic method of differential inequalities for this class of problems.

Among the important applications of asymptotic methods, we single out the development of the concept of asymptotic solution of inverse problems for nonlinear singularly perturbed reaction-diffusionadvection-type equations. A specific feature of mathematical models of this type is the presence in their solutions of stationary or moving narrow interior transition layers, which leads to instability in the numerical solution of direct problems and significantly complicates the further study of the corresponding inverse problems. The main idea of the concept of asymptotic solution of inverse problems is that asymptotic analysis makes it possible to reduce the original nonlinear singularly perturbed problem to a set of simpler problems that do not contain small parameters and have a lower spatial dimension or contain algebraic rather than differential equations. Thus, the use of asymptotic analysis makes it possible to establish simpler relationships between the observed parameters of the solution and the parameters of the inverse problem to be determined (coefficients in the equation, boundary and initial conditions, etc.), which can fundamentally change the approach to solving inverse problems. The presence of a small parameter in the original model provides in this case a sufficiently accurate qualitative and quantitative description of the solution. Recently, results have been obtained on the solution of inverse problems for a new class of nonlinear singularly perturbed Burgers-type equations with modular and quadratic advections with time-periodic coefficients, as well as some classes of initial-boundary value problems. It has been shown that, in such models, under certain conditions, the asymptotic approach makes it possible to reduce the coefficient inverse problems to linear algebraic equations relating, with a given accuracy, the observed position of the moving front with the input data of the model (coefficients in the equation and boundary condi- 
tions). Thus, if there is a possibility to observe the trajectory of the front on a certain time interval, then the question of determining the unknown coefficient of the equation or the boundary regime reduces to a set of simple algebraic operations. An asymptotic solution was proposed for the problem of recovery of the source function in the Burgers equation with modular advection from known (exactly or approximately) information about the trajectory of the front on a given time interval (period). In a similar manner, the asymptotic solution of the boundary control problem, i.e., determining the boundary conditions under which the front moves according to a given law, was obtained. The approach developed was demonstrated by solving the inverse linear gain coefficient problem on the basis of asymptotic analysis and efficient numerical methods in the case when the linear gain is obtained by solving an ill-posed problem of differentiating the observed information. The high efficiency of the approach proposed in comparison with those previously developed for such classes of problems was demonstrated (see [84-87]).

In a number of works, a new approach has been developed for the analytico-numerical study of moving fronts in singularly perturbed reaction-diffusion-advection-type models. A method for generating a dynamically adapted mesh for efficient numerical solution of problems of this class was proposed. The method is based on a priori information about the motion and properties of the front, obtained as a result of a rigorous asymptotic analysis of a singularly perturbed parabolic problem. In particular, the essential parameters taken into account when constructing the mesh are estimates of the position of the transition layer and its width and structure. The analytico-numerical approach proposed can significantly save computing resources, reduce the computation time, and increase the stability of the computing process in comparison with the classical approaches. Examples demonstrating the main ideas and techniques of using the proposed approach were considered (see, e.g., [88] and references therein).

The abilities of the methods of asymptotic analysis in solving the problem of diagnosing the destruction in time and space of a solution of the Burgers equation with quadratic and modular nonlinearities and cubic amplification were demonstrated (see [54]). The result obtained by the methods of asymptotic analysis was refined using the numerical diagnostics of the destruction of the exact solution, based on calculating an a posteriori asymptotically exact estimate of the error obtained when calculating the approximate solution on a sequence of condensing meshes. Numerical experiments demonstrate that the asymptotic result gives a good estimate for the time of destruction of the solution and its localization in space for a fairly wide range of small parameters. Thus, it can be stated that a new method has been created and is being developed for the analytical study of the phenomena of destruction of solutions of singularly perturbed problems (for example, problems for a Burgers-type equation). An important difference between this method and those used by other researchers is that it not only can establish the fact of the destruction of the solution in time (as well as to obtain an asymptotic approximation of the destruction time), but also to determine the spatial localization of this phenomenon, which is an important result for many applied problems. Below, the ideas of the asymptotic method of differential inequalities are demonstrated in two classes of relevant problems. These sections are now being actively developed and transferred to new classes of problems of both theoretical and applied interest.

\subsection{Multidimensional Interior Layers in a Problem with Balanced Nonlinearity}

In this section, we will illustrate the general scheme of using the asymptotic method of differential inequalities on the example of the problem of a multidimensional stationary contrast structure. This problem arises in the mathematical modeling of various processes with interphase transitions. tions:

Consider a spatially inhomogeneous reaction-diffusion equation with the Dirichlet boundary condi-

$$
\begin{gathered}
\frac{\partial u}{\partial t}=\epsilon^{2} \Delta u-f(u, x, \epsilon), \quad x \in \mathscr{D} \subset R^{N}, \quad t>0, \\
u(x)=g(x), \quad x \in \partial \mathscr{D}, \quad t>0, \\
u(x, 0, \varepsilon)=u^{0}(x, \varepsilon)
\end{gathered}
$$

and study the existence and Lyapunov stability of a stationary contrast structure. We will assume to be satisfied the following conditions:

(A1) The degenerate equation $f(u, x, 0)=0$ has exactly three roots $u=\varphi^{( \pm)}(x)$ and $\varphi^{(0)}(x)$, such that

$$
\varphi^{(-)}(x)<\varphi^{(0)}(x)<\varphi^{(+)}(x), \quad x \in \overline{\mathscr{D}}
$$


and

$$
\bar{f}_{u}^{( \pm)}(x) \equiv f_{u}\left(\varphi^{( \pm)}(x), x, 0\right)>0, \quad x \in \overline{\mathscr{D}}
$$

We introduce into consideration the function $I(x)$ :

$$
I(x):=\int_{\varphi^{(-)}(x)}^{\varphi^{(+)}(x)} f(u, x, 0) d u
$$

and suppose that

(A2) $I(x) \equiv 0, x \in \bar{D}$.

Under condition (A2), the equation in problem (18) is called an equation with balanced nonlinearity.

(A3) Let the boundary condition belong to the influence domain of the root $u=\varphi^{(-)}(x)$, i.e., the condition

$$
\int_{\varphi^{(-)}(x)}^{g(x)} f(u, x, 0) d u>0
$$

is satisfied for all $x \in \partial \mathscr{D}$.

We introduce the set of hypersurfaces

$$
\mathscr{S}=\{\Gamma \in \mathscr{D} \mid \Gamma \text { is an }(N-1) \text {-dimensional smooth connected closed manifold }\} .
$$

The asymptotics of the solution with an interior transition layer localized near some hypersurface $\Gamma_{\varepsilon}$ belonging to the set $\mathscr{S}$ is constructed according to the scheme described in the previous section. In this case, conditions $(A 1)$ and $(A 3)$ ensure the solvability of the problems for determining the leading terms of asymptotic representations (5) and (7). The leading terms of the regular parts of these representations are determined from the degenerate equation, and those of the boundary-layer parts are determined from standard boundary-value problems for autonomous second-order ODEs, the solvability of which is established using analysis on the phase plane (see, e.g., [20]). The operators $L_{R}^{ \pm}=-f_{u}\left(\varphi^{( \pm)}(x)\right)<0$ for $x \in \overline{\mathscr{D}}$. Therefore, they are invertible and, moreover, make it possible to construct lower and upper solutions of the required order by modifying the regular part of the asymptotics (see the previous section). The operator generating the boundary layer part of the asymptotic expansion,

$$
L_{B L}=\frac{\partial^{2}}{\partial \rho^{2}}-f_{u}\left(\varphi^{(-)}(x)+\Pi_{0}, x, 0\right), \quad x \in \partial \mathscr{D},
$$

where $\rho$ is the stretched distance along the normal to the boundary $\partial \mathscr{D}$, is well studied and, in particular, the corresponding inequality has a positive (exponentially decreasing) solution in the case of a monotonic boundary function $\Pi_{0}(\rho)$, the addition of which to the boundary-layer part of the asymptotics makes it possible to achieve the ordering of the lower and upper solutions in the boundary-layer zone (see [20]).

We define a function $V_{1}(x, \Gamma)$ for surfaces $\Gamma \in \mathscr{Y}$ as follows:

$$
V_{1}(x, \Gamma) \equiv-\kappa(x, \Gamma) m(x)+J(x ; \Gamma),
$$

where $\kappa(x, \Gamma)$ is the mean curvature of the surface $\Gamma$,

$$
\begin{gathered}
m(x)=\int_{-\infty}^{\infty}\left(\frac{\partial \tilde{Q}_{0}(\tau ; x)}{\partial \tau}\right)^{2} d \tau, \\
J(x ; \Gamma)=\int_{-\infty}^{\infty}\left[\tau\left(\left.\nabla_{x} f(u, x, 0)\right|_{u=\tilde{Q}_{0}(\tau ; x)} v(x ; \Gamma)\right)+f_{\varepsilon}\left(\tilde{Q}_{0}(\tau ; x), x, 0\right)\right] \frac{\partial \tilde{Q}_{0}(\tau ; x)}{\partial \tau} d \tau, \quad x \in \Gamma,
\end{gathered}
$$


$v(x ; \Gamma)$ is the unit normal vector to the curve $\Gamma$, directed to $\mathscr{D}^{(+)}, \tilde{Q}_{0}(\tau ; x)$ is the solution of the boundary value problem describing the interior transition layer in the zeroth approximation:

$$
\begin{array}{ll}
\frac{d^{2} \tilde{Q}_{0}}{d \tau^{2}}-f\left(\tilde{Q}_{0}, x, 0\right)=0, & \tau \in R, \\
\lim _{\tau \rightarrow \pm \infty} \tilde{Q}_{0}(\tau ; x)=\varphi^{( \pm)}(x), \quad \tilde{Q}_{0}(0 ; x)=\varphi^{(0)}(x), \quad x \in \Gamma,
\end{array}
$$

and $\tau$ is the stretched variable of the transition layer. It is known that this problem has a unique solution.

The $C^{1}$-matching condition (10) is satisfied in the first approximation if we impose the following requirement (note that, in the case of balanced nonlinearity, condition (10) is satisfied in the zeroth approximation for any surface $\Gamma$ ):

(A4) There is a surface $\Gamma_{0} \in \mathscr{Y}$ such that

$$
V_{1}\left(x, \Gamma_{0}\right) \equiv 0, \quad x \in \Gamma_{0} .
$$

As noted in the previous section, the $C^{1}$-matching condition (10) in the following approximations leads to linear equations for determining the coefficients $R_{i}$ in the asymptotic representation (3) for the transition surface $\Gamma_{\varepsilon}$ :

$$
A^{\Gamma} R_{i}=H_{i}
$$

where the operator $A^{\Gamma}$ is expressed via the original data as follows:

$$
\begin{gathered}
\mathscr{A}^{\Gamma} R(x):=m(x)\left(\Delta^{\Gamma}+\sum_{j=1}^{N-1} \kappa_{j}(x)^{2}\right) R(x)+\nabla_{\Gamma} m(x) \cdot \nabla_{\Gamma} R(x)-\kappa(x ; \Gamma) \frac{\partial m(x)}{\partial v(x ; \Gamma)} R(x)+J_{r}(x ; \Gamma) R(x) \\
=\operatorname{div}_{\Gamma}\left(m(x) \nabla_{\Gamma} R(x)\right)+\left(\sum_{j=1}^{N-1} \kappa_{j}(x)^{2}\right) R(x)-\kappa(x ; \Gamma) \frac{\partial m(x)}{\partial v(x ; \Gamma)} R(x)+J_{r}(x ; \Gamma) R(x), \quad x \in \Gamma
\end{gathered}
$$

where $\Delta^{\Gamma}, \operatorname{div}_{\Gamma}$, and $\nabla_{\Gamma}$ denote, respectively, the Laplace-Beltrami, divergence, and gradient operators on the surface $\Gamma_{0}$ and $\kappa_{j}(x)(j=1,2, \ldots, N-1)$ are the principal curvatures of $\Gamma_{0}$ at a point $x$. The function $J_{r}(x ; \Gamma)$ is defined as follows:

$$
\left.J_{r}(x ; \Gamma) \equiv \frac{d}{d r} J\left(x+r v(x ; \Gamma) ; \Gamma_{r}\right)\right|_{r=0} .
$$

The operator $A^{\Gamma}$ is self-adjoint, and all of its eigenvalues are real. Our next requirement is as follows.

(A5) The principal (maximum) eigenvalue $\lambda_{0}$ of the operator $A^{\Gamma}$ satisfies the condition $\lambda_{0}<0$.

It ensures the unique solvability of the problems for determining $R_{i}$, as well as the fulfillment of the condition for the existence of a positive solution of the inequality $A^{\Gamma} R<0$, which, as noted in Section 2.2, allows one to construct the lower and upper solutions, ordered in the zone of the transition layer. Thus, using the asymptotic method of differential inequalities, we obtain the following result for problem (18).

Theorem 1. Suppose that conditions $(A 1)-(A 5)$ are satisfied. Then, for sufficiently small $\varepsilon$, there exists a stationary solution $u(x, \varepsilon)$ of problem $(18)$, such that, outside any arbitrarily small but fixed neighborhoods $\Gamma_{\delta}$ of the surface $\Gamma_{0}$ and $\partial \mathscr{D}_{\delta}$ of the boundary $\partial \mathscr{D}$, there is a uniform passage to the limit

$$
\lim _{\varepsilon \rightarrow 0} u(x, \varepsilon)= \begin{cases}\varphi^{(-)}(x), & x \in \mathscr{D}^{(-)} \backslash \Gamma_{\delta} \backslash \partial \mathscr{D}_{\delta}, \\ \varphi^{(+)}(x), & x \in \mathscr{D}^{(+)} \backslash \Gamma_{\delta},\end{cases}
$$

where $\mathscr{D}^{(+)}$and $\mathscr{D}^{(-)}$are the internal and external (with respect to the surface $\Gamma_{0}$ ) subdomains of $\mathscr{D}$. This solution is locally unique and asymptotically stable with a stability region $\left[\alpha_{3}(x, \varepsilon), \beta_{3}(x, \varepsilon)\right]$. 


\subsection{Periodic Fronts in the Problem with Modular Advection}

Let us illustrate the development of an important line of research in problems with discontinuous coefficients and source terms by the following problem:

$$
\begin{gathered}
N_{\varepsilon}(u):=\varepsilon \frac{\partial^{2} u}{\partial x^{2}}-\frac{\partial u}{\partial t}+\frac{\partial|u|}{\partial x}-B(u, x, t)=0, \\
(x, t) \in \mathscr{D}:=\left\{(x, t) \in R^{2}:-1<x<1, t \in R\right\}, \\
u(-1, t, \varepsilon)=u^{(-)}(t), \quad u(1, t, \varepsilon)=u^{(+)}(t), \quad t \in R, \\
u(x, t, \varepsilon)=u(x, t+T, \varepsilon), \quad t \in R, \quad-1 \leq x \leq 1,
\end{gathered}
$$

for $\varepsilon \in I_{\varepsilon_{0}}:=\left\{0<\varepsilon \leq \varepsilon_{0}\right\}, 0<\varepsilon_{0}$. The functions $B, u^{(-)}$, and $u^{(+)}$are sufficiently smooth and $T$-periodic in $t$.

We define the regions $\mathscr{D}:=(x, t) \in(0 ; 1) \times(0, T], \quad \mathscr{D}_{T}^{(-)}:=(x, t) \in\left(0 ; x^{*}\right) \times(0, T], \quad$ and $\mathscr{D}^{(+)}:=(x, t) \in\left(x^{*} ; 1\right) \times(0, T]$ and give the following definition of the solution.

Definition. A function $u(x, t, \varepsilon) \in C(\overline{\mathscr{D}}) \cap C^{1} \mathscr{D} \cap C^{1,2}\left(\mathscr{D}^{(-)} \cup \mathscr{D}^{(+)}\right)$is called a solution of problem (19) if it satisfies the equation in (19) in each $D_{T}^{(\mp)}$ and the boundary conditions.

$\left(A_{0}\right)$ Suppose that $B, u^{(-)}$, and $u^{(+)}$are functions sufficiently smooth and $T$-periodic in $t$. Let $u^{(-)}(t)<0$ and $u^{(+)}(t)>0$ for $t \in R$.

For $\varepsilon=0$ in (19), we obtain the so-called degenerate equation

$$
\frac{\partial|u|}{\partial x}-\frac{\partial u}{\partial t}-B(u, x, t)=0
$$

We consider Eq. (20) with one of the additional conditions of problem (19):

$$
\begin{gathered}
u(-1, t)=u^{(-)}(t), \quad t \in R, \\
u(1, t)=u^{(+)}(t), \quad t \in R .
\end{gathered}
$$

Suppose to be satisfied the following conditions.

$\left(A_{1}\right)$ Problems (20), (21) and (20), (22) have solutions $u=\varphi^{(-)}(x, t)$ and $u=\varphi^{(+)}(x, t)$, defined at $(x, t) \in \overline{\mathscr{D}}, T$-periodic in $t$, and satisfying the condition

$$
\varphi^{(-)}(x, t)<0<\varphi^{(+)}(x, t), \quad(x, t) \in \overline{\mathscr{D}} .
$$

To formulate the following conditions, we introduce the function $I(x, t)$ as

$$
I(x, t):=\varphi^{(-)}(x, t)+\varphi^{(+)}(x, t),
$$

and require to be satisfied the following conditions.

$\left(A_{2}\right)$ The problem

$$
\frac{d x}{d t}=-\frac{I(x, t)}{\varphi^{(+)}(x, t)-\varphi^{(-)}(x, t)}, \quad x(t)=x(t+T),
$$

has a solution $x=x_{0}(t)$, satisfying the inequalities

$$
-1<x_{0}(t)<1 \quad \text { at } \quad t \in R
$$

$\left(A_{3}\right)$ The function

$$
K(x, t)=\frac{I(x, t)}{\varphi^{(+)}(x, t)-\varphi^{(-)}(x, t)} \quad \text { at } \quad x=x_{0}(t)
$$


satisfies the condition

$$
\int_{0}^{T} K_{x}\left(x_{0}(t), t\right) d t=: d_{0}>0 \quad \text { at } \quad t \in R .
$$

The position of the interior transition layer $x_{*}(t, \varepsilon)$ at each instant of time $t$ is defined as the point of intersection of the solution of problem (19) with the straight line $u=0$. The asymptotic approximation of the function $x_{*}(t, \varepsilon)$ is sought in the form of a series

$$
x_{*}(t, \varepsilon)=x_{0}(t)+\varepsilon x_{1}(t)+\ldots
$$

where $x_{k}(t), k=1,2, \ldots$ are $T$-periodic functions, which are determined from the condition of $C^{1}$-matching of the asymptotics of the problems $D_{T}^{(+)}$and $D_{T}^{(-)}$(these problems use an additional condition at the point $\left.x_{*}(t, \varepsilon): u\left(x_{*}(t, \varepsilon), t\right)=0\right)$. The formal asymptotics $U^{(\mp)}(x, t, \varepsilon)$ of the solution of each of these problems

$$
\begin{aligned}
& \varepsilon\left(\frac{\partial^{2} u}{\partial x^{2}}-\frac{\partial u}{\partial t}\right)+\frac{\partial|u|}{\partial x}-B(u, x, t)=0 \quad \text { for } \quad(x, t) \in \mathscr{D}^{(\mp)}, \\
& u(\mp 1, t, \varepsilon)=u^{(\mp)}(t), \quad u\left(x_{*}(t, \varepsilon), t, \varepsilon\right)=0 \quad \text { at } \quad t \in R, \\
& u(x, t, \varepsilon)=u(x, t+T, \varepsilon) \quad \text { at } \quad(x, t) \in \overline{\mathscr{D}}^{(\mp)}
\end{aligned}
$$

is sought in the form (according to the scheme of Section 2.1; for details, see, e.g., [68])

$$
U^{(\mp)}(x, t, \varepsilon)=\bar{U}^{(\mp)}(x, t, \varepsilon)+Q^{(\mp)}(\xi, t, \varepsilon)=\sum_{i=0}^{\infty} \varepsilon^{i}\left(\bar{U}_{i}^{(\mp)}(x, t)+Q_{i}^{(\mp)}(\xi, t)\right),
$$

where $\bar{U}_{i}^{(\mp)}$ and $Q^{(\mp)}$ denote the regular and boundary-layer (near $x_{*}$ ) parts of asymptotics (19).

The functions $x_{i}(t)$ are determined using the $C^{1}$-matching conditions

$$
\varepsilon \frac{\partial U^{(-)}}{\partial x}\left(x_{*}(t, \varepsilon), t, \varepsilon\right)=\varepsilon \frac{\partial U^{(+)}}{\partial x}\left(x_{*}(t, \varepsilon), t, \varepsilon\right), \quad t \in R
$$

in $i$ th order in $\varepsilon$. Using the standard procedure, we obtain a representation from which the coefficients of the regular part of asymptotics (24) are determined:

$$
\begin{gathered}
\varepsilon \frac{\partial^{2} \bar{U}^{( \pm)}}{\partial x^{2}}-\frac{\partial \bar{U}^{( \pm)}}{\partial t} \pm \frac{\partial \bar{U}^{( \pm)}}{\partial x}-B\left(\bar{U}^{( \pm)}, x, t\right)=0, \\
(x, t) \in \mathscr{D}^{( \pm)}, \quad u( \pm 1, t, \varepsilon)=u^{( \pm)}(t) \quad \text { at } \quad t \in R .
\end{gathered}
$$

Obviously, the leading terms of this representation, $\bar{U}_{0}^{( \pm)}$, are determined by condition $\left(A_{1}\right)$ and the higher orders terms are determined from the linear problems

$$
\begin{gathered}
\frac{\partial \bar{U}_{k}^{( \pm)}}{\partial t}, \quad \frac{\partial \bar{U}_{k}^{( \pm)}}{\partial x}+B_{u}\left(\varphi^{( \pm)}(x, t), x, t\right) \bar{U}_{k}^{( \pm)}=f_{k}^{( \pm)}(x, t) \\
\text { at } \quad(x, t) \in \mathscr{D}^{( \pm)}, \quad \bar{U}_{k}^{( \pm)}( \pm 1, t)=0 \quad \text { at } \quad t \in R,
\end{gathered}
$$

whose solution can be written out explicitly (it also implies the monotonicity of the operator generating the regular part of the asymptotics).

To construct the boundary layer part of asymptotics (24), we use the stretched variable $\xi=\left(x-x_{*}(t, \varepsilon)\right) / \varepsilon$, A.B. Vasilieva's representation of the nonlinearity of $B$, and the representation of the differential operator

$$
L_{\varepsilon}=\varepsilon \frac{\partial^{2}}{\partial x^{2}}-\frac{\partial}{\partial t}=\frac{1}{\varepsilon} \frac{\partial^{2}}{\partial \xi^{2}}+\frac{1}{\varepsilon} x_{*}^{\prime}(t, \varepsilon) \frac{\partial}{\partial \xi}-\frac{\partial}{\partial t} .
$$


For the coefficients of the boundary layer part of the asymptotics, we have the representation

$$
\begin{gathered}
\frac{1}{\varepsilon} \frac{\partial^{2} Q^{( \pm)}}{\partial \xi^{2}}+\frac{1}{\varepsilon} \frac{\partial x_{*}(t, \varepsilon)}{\partial t} \frac{\partial Q^{( \pm)}}{\partial \xi}-\frac{\partial Q^{( \pm)}}{\partial t}=\frac{1}{\varepsilon}\left[\mp \frac{\partial}{\partial \xi}\left(Q^{( \pm)}\right)\right] \\
+\left[B\left(\bar{U}^{( \pm)}\left(x_{*}(t, \varepsilon)+\varepsilon \xi, t, \varepsilon\right)+Q^{( \pm)}, x_{*}(t, \varepsilon)+\varepsilon \xi, t\right)\right. \\
\left.-B\left(\bar{U}^{( \pm)}\left(x_{*}(t, \varepsilon)+\varepsilon \xi, t, \varepsilon\right), x_{*}(t, \varepsilon)+\varepsilon \xi, t\right)\right], \\
Q^{( \pm)}(0, t, \varepsilon)+\bar{U}^{( \pm)}\left(x_{*}(t, \varepsilon), t, \varepsilon\right)=0 .
\end{gathered}
$$

From representation (26), we obtain equations $Q_{k}^{( \pm)}(\xi, t) \equiv Q_{k}^{( \pm)}\left(\xi, x_{*}(t, \varepsilon), t\right)$, for which we use the standard condition on $Q_{k}^{( \pm)}( \pm \infty, t)=0$. For $Q_{0}^{(-)}$and $Q_{0}^{(+)}$, we obtain the following problems:

$$
\begin{gathered}
\frac{\partial^{2} Q_{0}^{(-)}}{\partial \xi^{2}}=\left(1-x_{*}^{\prime}(t)\right) \frac{\partial Q_{0}^{(-)}}{\partial \xi} \quad \text { at } \quad \xi<0, \quad t \in R, \\
Q_{0}^{(-)}(-\infty, t)=0, \quad Q_{0}^{(-)}(0, t)=-\varphi^{(-)}\left(x_{*}(t), t\right), \quad t \in R,
\end{gathered}
$$

and

$$
\begin{gathered}
\frac{\partial^{2} Q_{0}^{(+)}}{\partial \xi^{2}}=\left(-1-x_{*}^{\prime}(t)\right) \frac{\partial Q_{0}^{(+)}}{\partial \xi}, \quad \xi>0, \quad t \in R, \\
Q_{0}^{(+)}(\infty, t)=0, \quad Q_{0}^{(+)}(0, t)=-\varphi^{(+)}\left(x_{*}(t), t\right), \quad t \in R .
\end{gathered}
$$

The solutions of problems (27), (28) and (29), (30) are written out explicitly: $Q_{0}^{(-)}(\xi, t)=-\varphi^{(-)}\left(x_{*}(t), t\right) \exp \left[\left(1-x_{*}^{\prime}(t)\right) \xi\right] \quad$ and $\quad Q_{0}^{(+)}(\xi, t)=-\varphi^{(+)}\left(x_{*}(t), t\right) \exp \left[\left(-1-x_{*}^{\prime}(t)\right) \xi\right] . \quad$ Obviously, $Q_{0}^{( \pm)}(\xi, t)$ is estimated as $\left|Q_{0}^{( \pm)}(\xi, t)\right| \leq c \exp (-\kappa|\xi|), \xi \in R^{( \pm)}, t \in R$. The $C^{1}$-matching condition (25) in the zeroth order,

$$
\frac{\partial Q_{0}^{(-)}}{\partial \xi}\left(0, x_{0}(t), t\right)=\frac{\partial Q_{0}^{(+)}}{\partial \xi}\left(0, x_{0}(t), t\right), \quad t \in R,
$$

is satisfied, since it reduces to the problem

$$
\begin{gathered}
\left(\frac{\partial Q_{0}^{(+)}}{\partial \xi}\left(0, x_{0}(t), t\right)-\frac{\partial Q_{0}^{(-)}}{\partial \xi}\left(0, x_{0}(t), t\right)\right)\left(\varphi^{(+)}\left(x_{0}(t), t\right)-\varphi^{(-)}\left(x_{0}(t), t\right)\right. \\
=\frac{d x_{0}}{d t}\left(\varphi^{(+)}\left(x_{0}(t), t\right)-\varphi^{(-)}\left(x_{0}(t), t\right)+I\left(x_{0}(t), t\right)=0\right.
\end{gathered}
$$

which is solvable by condition $\left(A_{2}\right)$. Note that this condition is analogous to the Rankine-Hugoniot condition for the class of problems under consideration.

From representation (26), one can obtain problems for $Q_{1}^{( \pm)}$and $Q_{k}^{( \pm)}$of the following orders, the solution of which can be written out explicitly. The $C^{1}$-matching condition (25) in the first order in $\varepsilon$ is reduced rather simply to the problem of finding a periodic solution of the equation

$$
\frac{d x_{1}}{d t}+K_{x}\left(x_{0}(t), t\right) x_{1}=\Phi_{1}(t)
$$

where $\Phi_{1}(t)$ are expressed via the known first-order asymptotic functions. The solution of this problem is guaranteed by condition $\left(A_{2}\right)$. The functions $x_{k}$ of the next orders are determined from similar problems, and condition $\left(A_{3}\right)$ is the condition for the unique solvability and monotonicity of the operator generating the asymptotics of the position of the interior transition layer.

We define $\mathscr{D}_{n}^{(-)}$and $\mathscr{D}_{n}^{(+)}$:

$$
\mathscr{D}_{n}^{(-)}:=\left\{(x, t) \in R^{2}:-1 \leq x \leq \sum_{i=0}^{n+1} x_{i}(t) \varepsilon^{i}, t \in R\right\},
$$




$$
\mathscr{D}_{n}^{(+)}:=\left\{(x, t) \in R^{2}: \sum_{i=0}^{n+1} x_{i}(t) \varepsilon^{i} \leq x \leq 1, t \in R\right\} .
$$

We denote by $U_{n}^{( \pm)}$partial sums of the $n$th order of representations (24), and $\xi$ is replaced with $\left(x-\sum_{i=0}^{n+1} x_{i}(t) \varepsilon^{i}\right) / \varepsilon$. We also introduce the notation

$$
U_{n}(x, t, \varepsilon):=\left\{\begin{array}{lll}
U_{n}^{(-)}(x, t, \varepsilon) & \text { at } & (x, t) \in \mathscr{D}_{n}^{(-)}, \\
U_{n}^{(+)}(x, t, \varepsilon) & \text { at } & (x, t) \in \mathscr{D}_{n}^{(+)}
\end{array}\right.
$$

Consider the initial-boundary value problem corresponding to problem (19):

$$
\begin{gathered}
\varepsilon\left(\frac{\partial^{2} v}{\partial x^{2}}-\frac{\partial v}{\partial t}\right)+\frac{\partial|v|}{\partial x}-B(v, x, t)=0, \\
(x, t) \in \mathscr{D}:=\left\{(x, t):-1<x<1, t \in R^{+}\right\}, \\
v(-1, t, \varepsilon)=u^{(-)}(t), \quad v(1, t, \varepsilon)=u^{(+)}(t) \quad \text { at } \quad t \in R^{+}, \\
v(x, 0, \varepsilon)=v^{0}(x, \varepsilon) \quad \text { at } \quad-1 \leq x \leq 1 .
\end{gathered}
$$

The proof of the existence and stability theorem for a periodic solution is conducted using the asymptotic method of differential inequalities, which, in this problem, uses the following definition of the lower and upper solutions.

Definition. Functions $\alpha$ and $\beta$ are called ordered lower and upper solutions of problem (19) for $\varepsilon \in I_{\varepsilon_{0}}$ if they satisfy the following conditions:

$$
\begin{array}{ll}
1^{\circ} & \alpha(x, t, \varepsilon) \leq \beta(x, t, \varepsilon) \quad \text { at } \quad(x, t) \in \overline{\mathscr{D}}, \\
2^{\circ} & N_{\varepsilon}(\alpha) \geq 0 \geq N_{\varepsilon}(\beta) \quad \text { at } \quad(x, t) \in \mathscr{D}^{+} \cup \mathscr{D}^{-}, \\
3^{\circ} & \alpha(-1, t, \varepsilon) \leq u^{(-)}(t) \leq \beta(-1, t, \varepsilon), \\
& \alpha(1, t, \varepsilon) \leq u^{(+)}(t) \leq \beta(1, t, \varepsilon) \quad \text { at } \quad t \in R .
\end{array}
$$

In the case when $\alpha$ and $\beta$ are continuous in $\overline{\mathscr{D}}$, but not smooth on some periodic curve $x=\bar{x}(t)$, they must have an admissible jump of the derivative:

$$
\begin{aligned}
& \frac{\partial \alpha}{\partial x}(\bar{x}(t)+0, t, \varepsilon) \geq \frac{\partial \alpha}{\partial x}(\bar{x}(t)-0, t, \varepsilon), \\
& \frac{\partial \beta}{\partial x}(\bar{x}(t)+0, t, \varepsilon) \leq \frac{\partial \beta}{\partial x}(\bar{x}(t)-0, t, \varepsilon) .
\end{aligned}
$$

Remark. It is known (see, e.g., [33]) that the existence of ordered lower and upper solutions implies the existence of a solution $u(x, t, \varepsilon)$ of problem (19), satisfying the inequalities

$$
\alpha(x, t, \varepsilon) \leq u(x, t, \varepsilon) \leq \beta(x, t, \varepsilon) \quad \text { at } \quad(x, t) \in \mathscr{D} \quad \text { and } \quad \varepsilon \in I_{\varepsilon_{0}} .
$$

In this problem, the lower and upper solutions are used with an admissible jump of the derivative on the modified curves of the formal asymptotics of the position of the interior layer $x_{\alpha}$ and $x_{\beta}$. In this case, there is the same result on existence (see, e.g., [36, 40], as well as [39] and references therein).

We have the following existence theorem for a solution of problem (19), its local uniqueness, and its asymptotic stability as a periodic solution of initial-boundary value problem (31).

Theorem 2. Let conditions $\left(A_{0}\right)-\left(A_{3}\right)$ be satisfied. Then, for sufficiently small $\varepsilon$, there exists a solution $u(x, t, \varepsilon)$ of problem (19) with a transition layer in the neighborhood $x_{0}(t)$, satisfying the limit relations

and the estimates

$$
\lim _{\varepsilon \rightarrow 0} u(x, t, \varepsilon)=\left\{\begin{array}{l}
\varphi^{(-)}(x, t) \quad \text { at } \quad-1 \leq x<x_{0}(t), \quad t \in R, \\
\varphi^{(+)}(x, t) \quad \text { at } \quad x_{0}(t)<x \leq 1, \quad t \in R,
\end{array}\right.
$$

$$
\left|u(x, t, \varepsilon)-U_{n}(x, t, \varepsilon)\right| \leq c \varepsilon^{n}, \quad(x, t) \in \bar{D}
$$


where $c$ is some positive constant independent of $\varepsilon$. This solution is asymptotically stable as a periodic solution of the initial-boundary value problem (31), with a stability region at least $\alpha_{2}(x, 0, \varepsilon) \leq u \leq \beta_{2}(x, 0, \varepsilon)$ and locally unique in this region as a solution of problem (19).

To prove the existence of a solution and to estimate the accuracy of the asymptotic approximation of the solution of problem (19), we use the lower and upper solutions $\alpha_{n}(x, t, \varepsilon)$ and $\beta_{n}(x, t, \varepsilon)$ constructed according to the scheme in Section 2.2. In this case, the modified asymptotics of the position of the transition layer, $x_{*}(t, \varepsilon)$, has the form

$$
\begin{aligned}
& x_{n \beta}(t, \varepsilon)=x_{0}(t)+\varepsilon x_{1}(t)+\ldots+\varepsilon^{n+1}\left(x_{n+1}(t)-\delta(t)\right), \\
& x_{n \alpha}(t, \varepsilon)=x_{0}(t)+\varepsilon x_{1}(t)+\ldots+\varepsilon^{n+1}\left(x_{n+1}(t)+\delta(t)\right) .
\end{aligned}
$$

In these modifications, used to construct the upper and lower solutions, the shift $\delta$ is determined from the periodic problem introduced in condition $\left(A_{3}\right)$ :

$$
\frac{d \delta(t)}{d t}+K_{x}\left(x_{0}(t), t\right) \delta(t)=h(t)
$$

where $h(t)$ is a periodic positive function. Therefore, $\delta>0$ due to the monotonicity of the operator of this problem. This ensures the necessary jump in the derivatives of $\alpha_{n}(x, t, \varepsilon)$ and $\beta_{n}(x, t, \varepsilon)$, as well as their ordering in the vicinity of the transition layer. The conditions of the definition are checked by the substitution into problem (19). The proof of the asymptotic stability of the periodic solution of problem (31) follows from the fact that the functions $\hat{\beta}(x, t, \varepsilon)$ and $\hat{\alpha}(x, t, \varepsilon)$ defined at $(x, t) \in \overline{\mathscr{D}}$ by the expressions

$$
\begin{aligned}
& \hat{\beta}(x, t, \varepsilon)= \begin{cases}u(x, t, \varepsilon)+\left(\beta_{2}^{(-)}(x, t, \varepsilon)-u(x, t, \varepsilon)\right) e^{-\varepsilon \gamma t}, & (t, x) \in \overline{\mathscr{D}}_{n \beta}^{(-)}, \\
u(x, t, \varepsilon)+\left(\beta_{2}^{(+)}(x, t, \varepsilon)-u(x, t, \varepsilon)\right) e^{-\varepsilon \gamma t}, & (t, x) \in \overline{\mathscr{D}}_{n \beta}^{(+)},\end{cases} \\
& \hat{\alpha}(x, t, \varepsilon)= \begin{cases}u(x, t, \varepsilon)+\left(\alpha_{2}^{(-)}(x, t, \varepsilon)-u(x, t, \varepsilon)\right) e^{-\varepsilon \gamma t}, & (t, x) \in \overline{\mathscr{D}}_{n \alpha}^{(-)}, \\
u(x, t, \varepsilon)+\left(\alpha_{2}^{(+)}(x, t, \varepsilon)-u(x, t, \varepsilon)\right) e^{-\varepsilon \gamma t}, & (t, x) \in \overline{\mathscr{D}}_{n \alpha}^{(+)},\end{cases}
\end{aligned}
$$

where $u=u(x, t, \varepsilon)$ is a periodic solution of problem $(19), \beta_{2}^{( \pm)}(x, t, \varepsilon)$ and $\alpha_{2}^{( \pm)}(x, \varepsilon)$ are the upper and lower solutions of problem (19), and $\overline{\mathscr{D}}_{n \beta}^{( \pm)}$and $\overline{\mathscr{D}}_{n \alpha}^{( \pm)}$are the subdomains of $\overline{\mathscr{D}}$ lying to the right and left of the curves $x_{n \beta}(t, \varepsilon)$ and $x_{n \alpha}(t, \varepsilon)$, are upper and lower solutions of problem (31).

A similar proof scheme is used in other periodic problems cited above.

\section{CONCLUSIONS}

This review reflects the development of methods for asymptotic analysis of new classes of nonlinear singularly perturbed problems for parabolic and elliptic equations, including the so-called reaction-diffusion-advection equations with boundary and interior transition layers. These studies include both the development of methods for constructing formal asymptotic approximations (satisfying the problem in the residual) and a rigorous substantiation: the proof of the existence of a solution, an estimate of the accuracy of the asymptotic approximation constructed, and an analysis of the Lyapunov stability of the solutions. The novelty of each class of problems can be determined by new differential operators generating asymptotics. The rigorous mathematical results presented in the article are based on the use of the asymptotic method of differential inequalities. The essence of this method is to use asymptotics to construct comparison functions: lower and upper solutions. In this case, the properties of operators generating asymptotics-invertibility and monotonicity - are revealed. This method determines the research strategy. The transition to new classes of problems, including the generalization of a number of results to the multidimensional case, as well as to some classes of systems important for applications, requires the development of both methods for constructing asymptotics and the study of the invertibility of new classes of operators generating asymptotics, and the study of their monotonicity. In a number of cases, this also required the use and modification of basic comparison theorems that establish conditions for the existence of the corresponding classes of solutions.

The results of asymptotic analysis obtained are widely used and form a basis both for the development of the theory of numerical methods and for the creation of efficient numerical algorithms for the study of problems with transition layers. In the course of work in this direction, a new method for the analytical 
study of the phenomena of destruction of solutions was created and is being developed, the field of which is the class of singularly perturbed problems (e.g., problems for a Burgers-type equation).

It is known that the dynamics of solutions of initial-boundary value problems for parabolic equations depends on the set of unstable stationary solutions. It is of interest to develop methods for proving the existence and finding instability conditions in the classes of problems presented above. Advances in this direction are presented in [89].

Among the important applications, we note the development of the concept of asymptotic solution of inverse problems for nonlinear singularly perturbed equations of the reaction-diffusion-advection type. It is known that the presence of stationary or moving narrow interior transition layers in their solutions leads to instability in the numerical solution of direct problems and significantly complicates the further study of the corresponding inverse problems. The main idea of the concept of asymptotic solution of inverse problems is that asymptotic analysis allows one to reduce the original nonlinear singularly perturbed problem to a set of simpler problems that do not contain small parameters and have a lower spatial dimension or contain algebraic rather than differential equations. Thus, the use of asymptotic analysis makes it possible to establish simpler relationships between the observed parameters of the solution and the parameters of the inverse problem to be determined (coefficients in the equation, boundary and initial conditions, etc.), which can fundamentally change the approach to solving inverse problems.

The results of asymptotic analysis obtained, the development of numerical methods and methods for solving inverse problems were applied to specific mathematical models of nonlinear wave theory, biology, magnetohydrodynamics, and urbo-ecological models. Among the important achievements, we note the creation of a model for the development of urban ecosystems, according to which the urban ecosystem can be regarded as an active environment with interacting natural and anthropogenic subsystems.

\section{ACKNOWLEDGMENTS}

The main part of the work is devoted to the ideas and results of the author, his disciples, and colleagues working at the Department of Mathematics of the Faculty of Physics of the Lomonosov Moscow State University. I am grateful to them. The results obtained create several new directions in nonlinear problems with interior and transition layers and, in our opinion, determine the world leadership in this area of research of A.N. Tikhonov's school on the singular perturbation theory. I am especially grateful to V.F. Butuzov for close scientific cooperation over many years.

\section{FUNDING}

This work was supported by the Russian Foundation for Basic Research, project no. 20-11-50080.

\section{OPEN ACCESS}

This article is licensed under a Creative Commons Attribution 4.0 International License, which permits use, sharing, adaptation, distribution and reproduction in any medium or format, as long as you give appropriate credit to the original author(s) and the source, provide a link to the Creative Commons license, and indicate if changes were made. The images or other third party material in this article are included in the article's Creative Commons license, unless indicated otherwise in a credit line to the material. If material is not included in the article's Creative Commons license and your intended use is not permitted by statutory regulation or exceeds the permitted use, you will need to obtain permission directly from the copyright holder. To view a copy of this license, visit http://creativecommons.org/licenses/by/4.0/.

\section{REFERENCES}

1. A. N. Tikhonov, "On the dependence of the solutions of differential equations on a small parameter," Mat. Sb. 22(64) (2), 193-204 (1948).

2. A. N. Tikhonov, "On systems of differential equations containing parameters," Mat. Sb. 27(69) (1), 147-156 (1950).

3. A. N. Tikhonov, "Systems of differential equations with small parameters multiplying derivatives," Mat. Sb. 31(73) (3), 575-586 (1952).

4. A. B. Vasil'eva and V. F. Butuzov, Asymptotic Methods in the Theory of Singular Perturbations (Vysshaya Shkola, Moscow, 1990) [in Russian].

5. P. Bates, D. Hilhorst, H. Matano, and Y. Morita, "Discrete and continuous dynamical systems," 37 (2), 1-3 (2017). 
6. M. Kumar and A. K. Singh, "Singular perturbation problems in nonlinear elliptic partial differential equations: A survey,” Int. J. Nonlinear Sci. 17 (3), 195-214 (2014).

7. K. Sakamoto, "Infinitely many fine modes bifurcating from radially symmetric internal layers," Asymptot. Anal. 42, 55-104 (2005).

8. R. Magnus, "The implicit function theorem and multi-bump solutions of periodic partial differential equations,” Proc. R. Soc. Edinburgh Sect. 136, 559-583 (2006).

9. M. del Pino, M. Kowalczyk, and J. Wei, "Resonance and interior layers in an inhomogeneous phase transition model," SIAM J. Math. Anal. 38, 1542-1564 (2007).

10. S. A. Kashchenko, "Dynamics of the logistic equation with two delays," Differ. Equations 52 (5), 538-548 (2016).

11. S. D. Glyzin, A. Yu. Kolesov, and N. Kh. Rozov, "Relaxation self-oscillations in Hopfield networks with delay," Izv. Math. 77 (2), 271-312 (2013).

12. N. Kopteva and E. O' Riordan, "Shishkin meshes in the numerical solution of singularly perturbed differential equations,” Inter. J. Numer. Anal. Model. 1 (1), 1-18 (2004).

13. N. Kopteva and M. Stynes, "Stabilized approximation of interior-layer solutions of a singularly perturbed semilinear reaction diffusion problem," Numer. Math. 119 (2), 787-810 (2011).

14. E. O'Riordan and J. Quinn, "Numerical method for a nonlinear singularly perturbed interior layer problem," Lect. Notes Comput. Sci. Eng. 81, 187-195 (2011).

15. J. Quinn, "A numerical method for a nonlinear singularly perturbed interior layer problem using an approximate layer location," Comput. Appl. Math. 290 (15), 500-515 (2015).

16. S. Franz and H.-G. Roos, "The capriciousness of numerical methods for singular perturbations," SIAM Rev. 53, 157-173 (2011).

17. S. Fran and N. Kopteva, "Green's function estimates for a singularly perturbed convection-diffusion problem," J. Differ. Equations 252, 1521-1545 (2012).

18. N. Kopteva, "Numerical analysis of a $2 \mathrm{~d}$ singularly perturbed semilinear reaction-diffusion problem," Lect. Notes Comput. Sci. 5434, 80-91 (2009).

19. V. F. Butuzov, A. B. Vasil'eva, and N. N. Nefedov, "Asymptotic theory of contrasting structures: A survey," Autom. Remote Control 58 (7), 1068-1091 (1997).

20. A. B. Vasil'eva, V. F. Butuzov, and N. N. Nefedov, "Contrast structures in singularly perturbed problems," Fundament. Prikl. Mat. 4 (3), 799-851 (1998).

21. V. F. Butuzov, N. N. Nefedov, and K. R. Schneider, "Singularly perturbed problems in case of exchange of stabilities," J. Math. Sci. 121 (1), 1973-2079 (2004).

22. A. B. Vasil'eva, V. F. Butuzov, and N. N. Nefedov, "Singularly perturbed problems with boundary and internal layers," Proc. Steklov Inst. Math. 268, 258-273 (2010).

23. P. C. Fife, "Semilinear elliptic boundary value problems with small parameters," Arch. Ration. Mech. Anal. 52, 205-232 (1973).

24. P. C. Fife and W. M. Greenlee, "Interior transition layers for elliptic boundary value problems with a small parameter," Russ. Math. Surv. 29 (4), 103-131 (1974).

25. N. N. Nefedov, "Method of differential inequalities for some classes of singularly perturbed partial differential equations,” Differ. Uravn. 31 (4), 719-723 (1995).

26. N. N. Nefedov, "Method of differential inequalities for some classes of nonlinear singularly perturbed problems with internal layers," Differ. Uravn. 31 (7), 1142-1149 (1995).

27. N. N. Nefedov, "An asymptotic method of differential inequalities for the investigation of periodic contrast structures: Existence, asymptotics, and stability,” Differ. Equations 36 (2), 298-305 (2000).

28. N. N. Nefedov and A. G. Nikitin, "The asymptotic method of differential inequalities for singularly perturbed integro-differential equations," Differ. Equations 36 (10), 1544-1550 (2000).

29. N. N. Nefedov, "Comparison principle for reaction-diffusion-advection problems with boundary and internal layers," Lect. Notes Comput. Sci. 8236, 62-72 (2013).

30. C. V. Pao, Nonlinear Parabolic and Elliptic Equations (Springer Science Business Media, New York, 1993).

31. M. G. Krein and M. A. Rutman, "Linear operators leaving invariant a cone in a Banach space," Usp. Mat. Nauk 3 (1), 3-95 (1948).

32. E. N. Dancer and P. Hess, "Behavior of a semi-linear periodic-parabolic problem when a parameter is small," Lect. Notes Math. 1450, 12-19 (1990).

33. P. Hess, Periodic-Parabolic Boundary Value Problems and Positivity (Pitman, New York, 1991).

34. P. P. Zabreiko, A. I. Koshelev, M. A. Krasnosel'skii, et al., Integral Equations: A Reference Text (Nauka, Moscow, 1967; Noordhoff, Leyden, 1975).

35. H. Amman, "On the existence of positive solutions of nonlinear elliptic boundary value problems," Indiana Univ. Math. J. 21, 125-146 (1971). 
36. D. H. Sattinger, "Monotone methods in nonlinear elliptic and parabolic boundary value problems," Indiana Univ. Math. J. 21 (11), 979-1001 (1972).

37. S. I. Pohozaev, "On equations of the form $\Delta u=(x, u, D u)$," Math. USSR-Sb. 41 (2), 269-280 (1982).

38. V. N. Pavlenko and O. V. Ul'yanova, "The method of upper and lower solutions for elliptic-type equations with discontinuous nonlinearities," Russ. Math. 42 (11), 65-72 (1998).

39. N. T. Levashova, N. N. Nefedov, O. A. Nikolaeva, A. O. Orlov, and A. A. Panin, "The solution with internal transition layer of the reaction-diffusion equation in case of discontinuous reactive and diffusive terms," Math. Methods Appl. Sci. 5134, 1-15 (2018).

40. V. N. Pavlenko and O. V. Ul'yanova, "Method of upper and lower solutions for parabolic-type equations with discontinuous nonlinearities," Differ. Equations 38 (4), 520-527 (2002).

41. C. De Coster, F. Obersnel, and P. A. Omari, "A qualitative analysis via lower and upper solutions of first order periodic evolutionary equations with lack of uniqueness," Handbook of Differential Equations: Ordinary Differential Equations (2006), Vol. 3, pp. 203-339.

42. V. N. Pavlenko, "Strong solutions of periodic parabolic problems with discontinuous nonlinearities," Differ. Equations 52 (4), 505-516 (2016).

43. R. Kannan and V. Lakshmikantham, "Existence of periodic solutions of semilinear parabolic equations and the method of upper and lower solutions," J. Math. Anal. Appl. 97 (1), 291-299 (1983).

44. P. Fife and M. Tang, "Comparison principles for reaction-diffusion systems: Irregular comparison functions and applications to question of stability and speed propagation of disturbances," J. Differ. Equations 40 (11), 168-185 (1981).

45. N. N. Nefedov, L. Recke, and K. R. Schnieder, "Existence and asymptotic stability of periodic solutions with an interior layer of reaction-advection-diffusion equations," J. Math. Anal. Appl. 405, 90-103 (2013).

46. N. T. Levashova, N. N. Nefedov, and A. O. Orlov, "Asymptotic stability of a stationary solution of a multidimensional reaction-diffusion equation with a discontinuous source," Comput. Math. Math. Phys. 59 (4), 573582 (2019).

47. N. N. Nefedov, A. G. Nikitin, M. A. Petrova, and L. Recke, "Moving fronts in integro-parabolic reaction-advection-diffusion equations," Differ. Equations 47 (9), 1318-1332 (2011).

48. N. N. Nefedov and K. Sakamoto, "Multi-dimensional stationary internal layers for spatially inhomogeneous reaction-diffusion equations with balanced nonlinearity," Hiroshima Math. J. 33 (3), 391-432 (2003).

49. A. O. Orlov, N. N. Nefedov, and N. T. Levashova, "Solution of contrast structure type for a parabolic reactiondiffusion problem in a medium with discontinuous characteristics," Differ. Equations 54 (5), 669-686 (2018).

50. N. T. Levashova, N. N. Nefedov, and O. A. Nikolaeva, "Solution with an inner transition layer of a two-dimensional boundary value reaction-diffusion-advection problem with discontinuous reaction and advection terms," Theor. Math. Phys. 207 (2), 655-669 (2021).

51. O. V. Rudenko, "Equation admitting linearization and describing waves in dissipative media with modular, quadratic, and quadratically cubic nonlinearities," Dokl. Math. 94 (3), 703-707 (2016).

52. O. V. Rudenko, “Modular solitons,” Dokl. Math. 94 (3), 708-711 (2016).

53. C. M. Hedberg and O. V. Rudenko, "Collisions, mutual losses, and annihilation of pulses in a modular nonlinear medium,” Nonlinear Dyn. 90, 2083-2091 (2017).

54. N. N. Nefedov and O. V. Rudenko, "On front motion in a Burgers-type equation with quadratic and modular nonlinearity and nonlinear amplification," Dokl. Math. 97 (1), 99-103 (2018).

55. N. N. Nefedov and O. V. Rudenko, "On the motion, amplification, and blow-up of fronts in Burgers-type equations with quadratic and modular nonlinearity," Dokl. Math. 102 (1), 283-287 (2020).

56. Yu. V. Bozhevol'nov and N. N. Nefedov, "Front motion in a parabolic reaction-diffusion problem," Comput. Math. Math. Phys. 50 (2), 264-273 (2010).

57. E. A. Antipov, N. T. Levashova, and N. N. Nefedov, "Asymptotics of the front motion in the reaction-diffusion-advection problem,” Comput. Math. Math. Phys. 54 (10), 1536-1549 (2014).

58. N. N. Nefedov, "Asymptotic analysis of reaction-diffusion-advection problems: Fronts with periodic motion and blow-up,” J. Phys. Conf. Ser. 811, 012008 (2017).

59. N. T. Levashova, N. N. Nefedov, and A. V. Yagremtsev, "Existence of a solution in the form of a moving front of a reaction-diffusion-advection problem in the case of balanced advection," Izv. Math. 82 (5), 984-1005 (2018).

60. N. D. Alikakos, P. W. Bates, and X. Chen, "Periodic traveling waves and locating oscillating patterns in multidimensional domains," Trans. Am. Math. Soc. 351 (7), 2777-2805 (1999).

61. A. I. Volpert and V. A. Volpert, Traveling Wave Solutions of Parabolic Systems (Am. Math. Soc., Providence, RI, 1994).

62. A. A. Samarskii, V. A. Galaktionov, S. P. Kurdyumov, and A. P. Mikhailov, Blow-up in Quasilinear Parabolic Equations (Walter de Gruyter, Berlin, 1995).

63. N. N. Nefedov and E. I. Nikulin, "Existence and stability of periodic contrast structures in the reaction-advection-diffusion problem,” Russ. J. Math. Phys. 22 (2), 215-226 (2015). 
64. N. N. Nefedov and E. I. Nikulin, "Existence and stability of periodic contrast structures in the reaction-advection-diffusion problem in the case of a balanced nonlinearity," Differ. Equations 53 (4), 516-529 (2017).

65. N. N. Nefedov and E. I. Nikulin, "Existence and asymptotic stability of periodic solutions of the reaction-diffusion equations in the case of a rapid reaction," Russ. J. Math. Phys. 25 (1), 88-101 (2018).

66. N. N. Nefedov and E. I. Nikulin, "Existence and asymptotic stability of periodic two-dimensional contrast structures in the problem with weak linear advection," Math. Notes 106 (5), 771-783 (2019).

67. N. N. Nefedov, E. I. Nikulin, and L. Recke, "On the existence and asymptotic stability of periodic contrast structures in quasilinear reaction-advection-diffusion equations," Russ. J. Math. Phys. 26 (1), 55-69 (2019).

68. N. N. Nefedov, "The existence and asymptotic stability of periodic solutions with an interior layer of burgers type equations with modular advection," Math. Model. Natural Phenomena 4 (4), 1-14 (2019).

69. V. F. Butuzov, N. N. Nefedov, L. Recke, and K. R. Schneider, "Periodic solutions with a boundary layer of reaction-diffusion equations with singularly perturbed Neumann boundary conditions," Int. J. Bifurcation Chaos 24 (8), 1440019-1-1440019-8 (2014).

70. N. N. Nefedov and E. I. Nikulin, "Periodic boundary layer solutions of a reaction-diffusion problem with singularly perturbed boundary conditions of the third kind," Differ. Equations 56 (12), 1594-1603 (2020).

71. N. N. Nefedov and N. N. Deryugina, "The existence of a boundary-layer stationary solution to a reaction-diffusion equation with singularly perturbed Neumann boundary condition," Moscow Univ. Phys. Bull. 75, 409414 (2020).

72. A. A. Melnikova, "Existence and stability of a front-type periodic solution of a two-component system of parabolic equations," Comput. Math. Math. Phys. 59 (7), 1131-1147 (2019).

73. A. A. Melnikova and N. N. Deryugina, "Existence of a periodic solution in the form of a two-dimensional front in a system of parabolic equations," Differ. Equations 56 (4), 462-477 (2020).

74. V. F. Butuzov and A. I. Bychkov, "Asymptotics of the solution of an initial-boundary value problem for a singularly perturbed parabolic equation in the case of double root of the degenerate equation," Differ. Equations 49 (10), 1261-1273 (2013).

75. V. F. Butuzov, N. N. Nefedov, L. Recke, and K. R. Schnieder, "On a singularly perturbed initial value problem in the case of a double root of the degenerate equation," Nonlinear Anal. Theory Methods Appl. 83, 1-11 (2013).

76. V. F. Butuzov, "Asymptotics of the solution of a system of singularly perturbed equations in the case of a multiple root of the degenerate equation," Differ. Equations, No. 2, 177-188 (2014).

77. V. F. Butuzov, "Asymptotic behavior of the solution to a singularly perturbed partially dissipative system with a multiple root of the degenerate equation," Sb. Math. 207 (8), 1100-1126 (2016).

78. V. F. Butuzov, "Asymptotic behavior and stability of solutions of a singularly perturbed elliptic problem with a triple root of the degenerate equation," Izv. Math. 81 (3), 481-504 (2017).

79. V. F. Butuzov, "On asymptotics for the solution of a singularly perturbed parabolic problem with a multizone internal transition layer," Comput. Math. Math. Phys. 58 (6), 925-949 (2018).

80. V. F. Butuzov, "Asymptotic expansion of the solution to a partially dissipative system of equations with a multizone boundary layer," Comput. Math. Math. Phys. 59 (10), 1672-1692 (2019).

81. V. F. Butuzov, "Asymptotic behavior of a boundary layer solution to a stationary partly dissipative system with a multiple root of the degenerate equation," Sb. Math. 210 (11), 1581-1608 (2019).

82. V. F. Butuzov, "On singularly perturbed systems of ODE with a multiple root of the degenerate equation," Izv. Math. 84 (2), 262-290 (2020).

83. V. F. Butuzov, "Asymptotics of a steplike contrast structure in a partially dissipative stationary system of equations," Comput. Math. Math. Phys. 61 (1), 53-79 (2021).

84. D. V. Lukyanenko, V. T. Volkov, N. N. Nefedov, and A. G. Yagola, "Application of asymptotic analysis for solving the inverse problem of determining the coefficient of linear amplification in Burgers' equation," Moscow Univ. Phys. Bull. 74, 131-136 (2019).

85. D. V. Lukyanenko, M. A. Shishlenin, and V. T. Volkov, "Asymptotic analysis of solving an inverse boundary value problem for a nonlinear singularly perturbed time-periodic reaction-diffusion-advection equation," J. Inverse Ill-Posed Probl. 27 (5), 745-758 (2019).

86. V. T. Volkov and N. N. Nefedov, "Asymptotic solution of coefficient inverse problems for Burgers-type equations," Comput. Math. Math. Phys. 60 (6), 950-959 (2020).

87. N. N. Nefedov and V. T. Volkov, "Asymptotic solution of the inverse problem for restoring the modular type source in Burgers' equation with modular advection," J. Inverse Ill-Posed Probl. 28 (5), 633-639 (2020).

88. V. T. Volkov, D. V. Lukyanenko, and N. N. Nefedov, "Analytical-numerical approach to describing time-periodic motion of fronts in singularly perturbed reaction-advection-diffusion models," Comput. Math. Math. Phys. 59 (1), 46-58 (2019).

89. O. E. Omel'chenko, L. Recke, V. F. Butuzov, and N. N. Nefedov, "Time-periodic boundary layer solutions to singularly perturbed parabolic problems," J. Differ. Equations 262 (9), 4823-4862 (2017).

Translated by E. Chernokozhin 